\title{
Cirugía, ética y bioética. Un llamado a la razón
}

\author{
Surgery, ethics and bioethics. A call to reason
}

Alejandro Weber Sánchez*

Palabras clave: Ética, bioética, cirugía.

Key words: Ethics, bioethics, surgery.
* Adscrito al

Departamento de Cirugía del Hospital Ángeles

Lomas. Profesor de Ética de la Salud, Facultad de Ciencias de la Salud.

Universidad Anáhuac.

Recibido: 09/06/2016 Aceptado: 07/09/2016

\section{RESUMEN}

La reflexión sobre las actitudes y motivos que mueven a los cirujanos son indispensables para la adecuada estrategia decisional. La búsqueda basada en la razón, los valores y la metodología que nos brinda la ética y bioética pueden ayudar a encontrar las respuestas adecuadas para decidir optando por el bien y lo correcto. Las nociones del marco antropológico adecuado, el bien, la finalidad y tener clara la jerarquía de valores ayudan al análisis.

\section{ABSTRACT}

The reflection on the attitudes and motives that move surgeons are indispensable for the adequate decision strategy. The search based on reason, values and methodology that ethics and bioethics gives us, can help us find the correct answers to take decisions opting for the good and correct ones. The notions of a proper anthropological framework, the good, the purpose and having a clear hierarchy of values, help the analysis.
$\mathrm{E}^{\mathrm{n}}$ n una época en que, al parecer, solamente la evidencia empírica tiene la fuerza que requiere cualquier afirmación, resulta no sólo útil sino necesario hacer una reflexión sobre las actitudes y motivos que nos mueven a los médicos $-\mathrm{y}$, particularmente, a los cirujanos- cuando la sociedad, e incluso las autoridades, se dan cuenta más que nunca que las consideraciones de tipo bioético son indispensables en la medicina y la práctica clínica. Recientemente, por disposición jurídica, los hospitales tienen obligación de contar con comités bioéticos que regulen algunas de las prácticas clínicas.

A casi 50 años de la aparición del término en el artículo de Van Rensselaer Potter, ${ }^{1}$ de la bioética se habla mucho: hay disponibles multitud de artículos, libros, cursos, maestrías y doctorados que pueden ser tomados incluso por la red; sin embargo, la ética y la bioética no son algo mágico que transforme con su sola mención la conducta en el sentido adecuado: son más bien herramientas que ayudan a dirigir las acciones para ser y hacer lo correcto. Primero, habría que hacer precisamente la reflexión sobre ese sentido correcto al cual deberían apuntar decisiones y actos biomédicos.
Por supuesto, partimos del hecho de que las personas tenemos una ética $y$, de acuerdo con esto, decidimos y actuamos en nuestra vida y nuestra profesión. En el caso especial del cirujano, se podría pensar que basta ser hábil y diestro en el arte de la cirugía, tener sentido común, experiencia clínica, ser una buena persona y tener buenas intenciones para actuar correctamente, pero estos factores, aunque importantes, no son una garantía de la respuesta y solución apropiada ante los múltiples dilemas éticos que enfrentamos en nuestra profesión; tampoco se puede confiar en que contar con códigos de conducta elaborados por las organizaciones especializadas es suficiente, a la manera de un vademécum, para saber cómo actuar en todo momento. Estas decisiones no se refieren a los juicios clínicos o biotecnológicos para los que estamos preparados y continuamos aprendiendo para brindar a los pacientes la mejor atención, con técnicas quirúrgicas modernas y mejorando las que ya conocemos. Se trata de los juicios acerca de lo correcto o incorrecto, cuestiones filosóficas profundas que, indiscutiblemente, provocan fuertes emociones en todos; opiniones personales arraigadas en las propias creencias y preferencias, que muchas veces involucran intereses individuales; 
todo ello, asuntos existenciales que requieren una adecuada estrategia decisional. ${ }^{2}$

Habría que hacer, como los griegos clásicos, una distinción entre lo que es la doxa (la opinión), que siempre es respetable por ser de las personas (aunque no por ello necesariamente tenga que ser atendible), y la aletheia, el conocimiento fundado en los hechos, la adecuación del intelecto a la realidad, conocida filosóficamente como verdad. ${ }^{3}$ Verdad que, aunque sólo podemos conocer parcialmente, de manera imperfecta y análoga, debería ser buscada siempre con base en la razón que tenemos el privilegio de poseer. ${ }^{4}$ Por ello, es necesario hacer un alto en el camino y buscar tanto en las raíces y valores familiares recibidos como en la filosofía moral - y, específicamente, en esa relativamente nueva ciencia llamada bioética - aquellas herramientas que nos puedan ayudar a encontrar el camino entre tantas encrucijadas que no están tan bien señaladas como deberían, o aún peor, que por el resultado conseguido en ocasiones, parecen haber tenido la señalización equivocada.

Hablar de la bioética sin hacer referencia a la ética es un sinsentido. La ética se encarga de resolver los problemas relativos a la conducta humana en cuanto a lo correcto o incorrecto, el bien y el mal (considerados como realidades objetivas), y no sólo en cuanto a las preferencias o creencias personales; de entrever las razones por las cuales una acción es correcta o incorrecta. Su papel se ha justificado desde que el ser humano se da cuenta de que, aunque todos los seres son como deben ser y actúan como deben actuar, responden a una naturaleza o modo de ser que no pueden evadir. Sin embargo, él es una excepción, pues se da cuenta de que sus actos dependen de su inteligencia racional y de tener una voluntad libre que lo faculta para actuar, en ocasiones incluso en contra de la razón o lo correcto. Son precisamente esas dos características las que lo convierten en responsable de sus acciones, en cuanto ser libre y no determinado; ésa es la grandeza y tragedia del ser humano, pues su actuar puede apoyarse más que en la recta razón, sólo en la corriente social, lo aprendido o sus preferencias o intereses, cualquiera que éstos sean. ${ }^{5}$

Íntimamente asociada con la noción de la ética, debe estar implicada la noción de la finalidad, pues a excepción de los fatalismos o determinismos, la filosofía enseña que todo en la naturaleza está orientado a un fin; no se trata más que de una de las aplicaciones trascendentales del principio metafísico de la finalidad. Fin es aquello por lo cual algo se crea, se intenta o se lleva a cabo. La naturaleza humana no es una excepción, y también está orientada a un fin, que es su desarrollo y plenitud; en otras palabras, que llegue a ser todo lo que esa naturaleza le permite llegar a ser, incluida su felicidad. ${ }^{6}$ Toda persona ansía la felicidad sin límite, lo cual explica los esfuerzos por evitar todo aquello que piensa que interfiere con su felicidad, aunque sea en forma transitoria.

En el caso del actuar humano, un fin es un bien que, una vez conocido, hace que la voluntad se dirija a actuar para conseguirlo; por lo tanto, de esos fines depende su actuar. Todo aquello por lo que actuamos es porque lo consideramos un bien, aunque nuestra inteligencia pueda estar errada por diferentes circunstancias. Y iqué es, entonces, el bien? Es todo aquello que va de acuerdo y conviene a nuestra naturaleza racional y la dignidad de la naturaleza humana. Así, nuestras acciones deberían apuntar a la felicidad perfecta que, de acuerdo a Boecio, es "un estado perfecto ocasionado por la reunión de todos los bienes, a lo cual todos deberíamos aspirar". ${ }^{7}$

Por ello, es conveniente distinguir diferentes aspectos de la finalidad para poder actuar mejor. La finalidad puede ser considerada en cuanto al origen de la obra, es decir, al bien al que la obra está orientada por su misma naturaleza, y que es diferente del fin que pueda tener el autor. En el caso específico de la cirugía, la colecistectomía, por ejemplo, tiene la finalidad de extirpar la vesícula cuando existe una patología que lo justifica, pero el cirujano puede tener la finalidad de extirparla aunque no se requiera para obtener una ganancia.

Es evidente que para que el acto sea correcto, tanto la obra en sí como la finalidad de quien la realiza deben ser buenas. También hay que considerar el fin de acuerdo con su dignidad. Puede ser deleitable si la acción se realiza para obtener placer, útil cuando el bien que intenta está encaminado a la obtención de otro mayor, y honesto cuando logra un bien que satisface las aspiraciones racionales y dignas que debe tener la persona humana. Es posible, por supuesto, 
que una acción combine estas tres dimensiones, como en el caso del cirujano que disfruta cuando opera, logra el fin del procedimiento y lo hace de manera honesta. Sería ideal que siempre fuera así; sin embargo, es evidente que lo más importante es que lo que hacemos sea honesto, aun cuando la acción no sea de nuestro entero gusto.

Por último, si lo consideramos en cuanto al intervalo, el fin próximo es aquel que se logra de inmediato, es intermedio cuando se logra como resultado del fin próximo, y último cuando es resultado de los anteriores y ya no está subordinado a otro fin superior en el mismo orden. Es frecuente para el hombre guiarse más por los fines próximos e intermedios y no por el fin último. Como hombres y cirujanos deberíamos preguntarnos más por nuestro fin último para conformar a éste todos los actos, aspiraciones y tendencias en nuestra vida, aunque esto represente en ocasiones refrenar los deseos más inmediatos y menos nobles. ${ }^{6}$

¿Qué es, entonces, la bioética? Aunque no hay una sola definición, es parte de la filosofía moral que considera lo lícito de los actos relacionados con la vida del hombre y, particularmente, con aquéllos vinculados con la práctica y desarrollo de las ciencias médicas y biológicas. ${ }^{8}$ La Encyclopedia of Bioethics la define como "estudio sistemático de la conducta humana en el ámbito de las ciencias de la vida y del cuidado de la salud examinada a la luz de los valores y de los principios morales". ${ }^{9}$ Analiza la incidencia humana sobre los cambios y fenómenos vitales para discernir las opciones más aceptables de acuerdo al marco antropológico del cual se parte, para determinar criterios y asignar los límites de la práctica médica y la investigación científica. ${ }^{10}$ No determina ni elabora nuevos principios éticos generales, sino que aplica los principios generales a los nuevos problemas que se ofrecen a la consideración en el reino de la vida. ${ }^{11}$ Su abordaje es multidisciplinar: no es sólo médico ni sólo científico. Se apoya en una diversidad de ciencias que, puestas en común, ayudan a una forma de conocimiento aplicado que se produce en y con el encuentro y participación de los diversos saberes, proporcionando una mejor forma de entender y abordar el fenómeno o la problemática determinada. ${ }^{12}$ No pretende ser una lista de principios o códigos de ética a aplicar ni tampoco lleva a obtener respuestas de validez universal, sino que ayuda a orientar las posturas correctas. Como expone Vila-Coro: "En las posturas éticas existe, implícita o explícitamente, una determinada concepción antropológica, y de esta visión del hombre derivan las posiciones éticas". ${ }^{13}$

La metodología de la bioética se asemeja al método científico ante el dilema bioético. Utiliza un análisis empírico, un examen de los hechos y la realidad utilizando ese enfoque interdisciplinario, no sólo como acumulación de diferentes saberes, sino como una apertura recíproca entre ellos, sin reduccionismos ni sectarismos que constriñan la complejidad del problema, sino para tener una mejor comprensión del hecho. Así, desde sus diferentes enfoques y una vez teniendo este conocimiento, lo somete a la concepción antropológica de la cual parte, confrontándola contra una jerarquía de valores; con ello, finalmente, determina la licitud de los actos. ${ }^{10}$ Tanto el marco antropológico como la jerarquía de valores pueden ser entendidos de manera diferente entre las personas, lo cual permite comprender mejor cómo pueden darse posturas bioéticas antitéticas. Por ello, la consideración de la persona pasa a ser el elemento fundamental para la evaluación de los actos bioéticos. Bastaría reflexionar sobre su esencia y valor especial por ser el único ser vivo dotado de inteligencia y voluntad libre, lo que lo convierte en el ente más valioso, por su dignidad y trascendencia. Entendida en su multidimensionalidad tanto física como psicológica, social y espiritual, dimensiones inseparables aunque manifestadas en forma diversa, cada persona debe ser considerada única, irrepetible, insustituible e indisponible, y por ello, centro y criterio para las consideraciones bioéticas.

El individuo tiene conciencia de sí mismo, por lo que puede conocer la realidad, aprehender y apreciar elementos incluso abstractos como los valores, y entender que requiere de normas tanto sociales como jurídicas para su sana convivencia y supervivencia. Pero también el hombre se ha dado cuenta desde la antigüedad de que hay otras normas que existen más allá de las que puede darse él mismo, como menciona Cicerón en De República: "hay una ley verdadera, recta razón conforme con la naturaleza, presente en todos, constante, sempiterna, la cual llama al deber mandando y desvía del 
engaño prohibiendo (...) A esta ley (...) ni es lícito derogarla en algo, ni puede ser abrogada por completo, ni nos podemos desligar de esta ley por obra del Senado o del Pueblo (...) no será una en Roma, otra en Atenas, una ahora, otra después; todos los pueblos, en todos los tiempos, serán regidos por esta única ley eterna e inmutable". ${ }^{14} \mathrm{~A}$ ésta se le ha llamado "ley natural", de la cual derivan los deberes y derechos para que pueda hacerse efectivamente el bien y evitar el mal, y es perceptible por la razón.

Es a partir de la diferente consideración que existe de lo que es la persona y de la jerarquía que se tiene de los valores, que se dan diferentes posturas bioéticas. Puede ser que la libertad, la opción autónoma del sujeto, sea ponderada como el valor supremo, o la utilidad que proporciona la acción o sus consecuencias, o la valoración de los cambios evolutivos en la sociedad, aceptando la transformación de los valores fundamentales y admitiendo los que los individuos y sociedad puedan seleccionar a su antojo como respuesta de su conformación al ambiente (como apunta Tarasco, "basado en [...] la prioridad de la especie respecto al individuo [...] y la evolución del comportamiento con los valores morales reconocidos, por los cuales el 'así es' es igual al 'así debe ser'"'). ${ }^{10}$ Otra postura es considerar valores reconocidos como fundamentales, como lo hace el principialismo de Beauchamp y Childress, fundando las decisiones en los principios de beneficencia, no maleficencia, justicia y autonomía, ${ }^{15}$ lo que constituye la principal corriente en los Estados Unidos.

Es indudable que tanto la libertad como la utilidad o los principios mencionados son valiosos y deben ser apreciados en cualquier corriente de pensamiento que tome en cuenta la ética. Sin embargo, si no está presente en la consideración la persona y su bien integral como prioridad fundamental, es mucho más fácil fallar en las decisiones bioéticas. En muchas ocasiones, aunque parezca absurdo, no se toman la vida y la dignidad de la persona como valores fundamentales, por lo que puede errarse al elegir cualquier otro valor por encima de ellas, cayendo en un relativismo donde la situación es el criterio principal y permite cambiar la ponderación. Ya expresaba Kant en la Metafísica de las Costumbres que "la humanidad misma es una dignidad, porque el hombre no puede ser tratado por ningún hombre como un simple instrumento, sino siempre como un fin; en ello estriba su dignidad." Por ello, el hombre es la fuente y el objeto de la moralidad. La corriente llamada "personalismo" defiende como prioritario el valor de la persona, fundado en su dignidad esencial y su plena realización.

Con la persona como centro y criterio, hay principios de moralidad universalmente válidos para todo ser humano y cultura, que sirven de guía para actuar. Aunque son de sentido común, vale la pena no sólo recordarlos sino hacerlos parte de los actos cotidianos; algunos que podríamos mencionar son: todo bien posible es obligatorio; hacer el bien y evitar el mal; actuar de tal modo que la acción pueda erigirse en norma universal de conducta; el fin nunca justifica los medios; no se deben buscar fines buenos que tengan efectos desproporcionadamente malos; es valioso todo aquello que contribuya al desarrollo auténtico de la persona; el bien no es menor porque pueda beneficiar a otros, ni el mal porque otro lo sufra; y es preferible el bien común al privado, si ambos son del mismo orden. ${ }^{16}$ El personalismo parte del concepto de que la verdad existe, es objetiva y por ello hay que buscarla. Reconoce en la persona la indivisibilidad de una unidad física, psíquica y trascendente, independientemente de su desarrollo físico o psicológico, de la condición de su existencia (salud o enfermedad), de las propiedades que posee o las capacidades que está en grado de adquirir. ${ }^{17}$ Es consciente de que la dignidad de la persona proviene de su esencia y que por esta naturaleza, todos los seres humanos merecemos el mismo respeto. ${ }^{18}$ De aquí parten principios particulares del personalismo, que es fundamental apreciar y practicar, como el valor fundamental de la vida física, valor supremo sobre el cual todo se subordina; el de la corporeidad, dado que el cuerpo no puede reducirse a un mero instrumento; la libertad responsable, que debe guiar los actos tanto del paciente como del médico; el principio de totalidad o terapéutico, fundado en el todo unitario de la corporeidad humana resultante de partes distintas y unificadas entre sí, orgánica y jerárquicamente por la existencia única y personal. ${ }^{8}$ Cualquier intervención en la vida de las personas tiene justificación si tiene 
un propósito terapéutico. Si el cuerpo es un todo unitario en la persona, toda intervención sobre una "parte" no puede prescindir de la consideración del "todo". En el fondo, como menciona Sgreccia, este principio rige toda la licitud y obligatoriedad de la terapia médica y quirúrgica; y el principio de socialidad, que busca el logro del bien de la comunidad a través del bien individual, que consiste en la promoción de la vida y salud de la sociedad a través de la promoción de la vida y salud de la persona singular; por último, el principio de subsidiariedad, integrado en el anterior, que requiere la obligación del cuidado de los más necesitados fundada en el reconocimiento de la dignidad de los demás en cuanto a personas: la persona es la fuente y el fin de la sociedad, en cuanto se realiza participando en la realización del bien de los semejantes. ${ }^{19}$

La bioética no puede brindar fórmulas extraordinarias para resolver los dilemas; no hay bioética que sea adecuada si no consideramos y cuidamos nuestra dignidad como personas y consideramos y protegemos la dignidad de las demás y cada una de las personas. Quizá, aunque parezca imposible en esta época, lo mejor es pensar que el amor a los semejantes puede ser la mejor guía, pues el verdadero amor no daña, no lastima, no ofende y no lesiona. Los médicos $-y$, en especial, los cirujanos - tenemos obligaciones éticas específicas que van más allá de las de otras personas o ciudadanos. Retomando el liderazgo histórico logrado a través de los siglos, los cirujanos tenemos un gran reto ante los problemas bioéticos que se nos presentan a diario y los que afronta la sociedad. Tenemos, además, la obligación de seguir formando nuestra conciencia para responder a estos retos cada vez más urgentes y difíciles; más aún cuando en ocasiones somos llamados a formar parte de comités de bioética, que son una gran responsabilidad. Siempre deberíamos tener claros los criterios para decidir lo que es el bien y recordar que somos custodios y servidores de las vidas humanas que tenemos en nuestras manos.

\section{REFERENCIAS}

1. Potter VR. Bioethics: the science of survival. Perspectives in Biology and Medicine. 1970; 14: 127-153.
2. Lo B. Resolving ethical dilemmas. A guide for clinicians. $5^{a}$ ed. Philadelphia: Lippincott Williams \& Wilkins; 2013. p. 4.

3. Ayala BF. La retórica de la opinión pública. Rev Mex Opinión Pública. 2009; 6: 73-79.

4. Juan Pablo II. Fe y razón. México: Ed. Paulinas; 1998. p. 93.

5. Lucas LR. Explícame la persona. Roma: Edizioni ART; 2010. p. 107.

6. Alvarado ME. Ética. México: Ed. Trillas; 2010. p. 17-18.

7. de Villegas EM. Las eróticas y traducción de Boecio. Tomo II. Madrid: 1774. p. 122. Disponible en: https://books.google.com.mx/books?id=NEvrP2UI2 $w 8 C \& p g=$ PA122\&dq = boecio + felicidad + perfecta $\& \mathrm{hl}=\mathrm{es} 419 \& \mathrm{sa}=$ X\&ved =0ahUKEwityei4lqjNAhUlc1l KHau1A6AQ6AEIHTAA\#v $=$ onepage $\& q=$ boecio $\% 20$ felicidad\%20perfecta\& $\mathrm{f}=$ false

8. Sgreccia E. Manual de bioética. México: Diana; 1996. p. 42.

9. Encyclopedia of Bioethics. Vol. I. New York: MacmiIlan; 1978. p. 19.

10. Tarasco MM. Diversas posturas filosóficas que influyen en el razonamiento bioético. En: Kuthy P, Villalobos $\mathrm{P}$, Martínez G, Tarasco M. Introducción a la bioética. $3^{\text {a }}$ ed. México: Mendez Ed.; 2009. p. 13-43.

11. Polaino-Lorente A. Manual de bioética general. $4^{\mathrm{a}}$ ed. Madrid: Rialp; 2000. p. 23.

12. Boeiro M, Estrada G. Interdisciplinariedad y multidisciplinariedad. Un ensayo en la enseñanza de las finanzas y administración pública. Argentina: Facultad de Ciencias Económicas. Universidad Nacional de Rio Cuarto; 1998. Disponible en: http://www. angrad.org.br/_resources/files/_modules/producao/ producao_715_201212051834228e9c.pdf.

13. Vila-Coro MD. La bioética en la encrucijada: sexualidad, aborto, eutanasia. Madrid: Dykinson; 2003.

14. Cicerón, De Legibus, XXI, 17 y De República III, 2. Disponible en: https://webcache.googleusercontent.com/search?q= cache:tfDwLDIITDAJ:ht tps://compendiodelcatecismo.com/2012/11/13/ ley-natura-un-invento- 1 a-parte $/+\& c d=1 \& \mathrm{hl}=$ es$419 \& \mathrm{ct}=\mathrm{clnk} \& \mathrm{gl}=\mathrm{mx}$.

15. Beauchamp TL, Childress JF. Principles of biomedical ethics. New York: Oxford University Press; 2009. p. 12-13.

16. Llano C. Dilemas éticos de la empresa contemporánea. México: FCE; 1997. p. 22.

17. Dàgostino F, Palazzani L. Bioetica: Nozzioni Fondamentali. Brescia: Editrice la Scuola; 2007.

18. Petrini C, Gainotti S. A personalist approach to publichealth ethics. Bull World Health Organ. 2008; 86: 624-629.

19. Palazzani L. La fundamentación personalista en bioética. Instituto di Bioetica. Cuadernos de Bioética Univeristà Cattolica S. Cuore Roma. 2004; 14 (2-93): p. 48-54.

\section{Correspondencia:}

Dr. Alejandro Weber Sánchez

Vialidad de la Barranca s/n Consultorio 410

Colonia Valle de las Palmas, 52787,

Huixquilucan, Edo. de México.

Tel: 52469527

E-mail: awebersanchez@gmail.com 